\title{
Investigation of piezoresistive sensor for robotic gripping operations
}

\begin{abstract}
Various types of tactile pressure sensors in robotic grasping operations have been effectively implemented in robotic hand fields. Some useful approaches of these tactile sensors are briefly discussed in this study based on their robotic hand applications. A robotic hand model is also designed to employ the tactile sensors that have recorded the information that is collected through the physical interaction between the pressure sensors and the object. This study has practically investigated the response of the piezoresistive pressure sensor during the object gripping operations in different weights. Empirical results have proven that the piezoresistive pressure sensor is the proper technique that can be implemented in robotic hand applications. The correlation between the input force and the output volt has been experimentally derived to achieve the efficient gripping operation.
\end{abstract}

Keyword: Piezoresistive pressure sensors; Robotic grasping; Robotic hand; Gripping 